\title{
LA TRIBUTACIÓN DE LAS PENSIONES PÚBLICAS*
}

\author{
The maintenance of public pensions
}

\author{
Miguel Gutiérrez Bengoechea \\ Profesor Titular de Derecho Financiero \\ Universidad de Málaga \\ mgutierrez@uma.es
}

doi: http://dx.doi.org/10.18543/ed-66(1)-2018pp171-196

Recibido: 12.04 .2018

Aceptado: 25.06.2018

\section{Resumen}

El envejecimiento poblacional es un problema demográfico de carácter estructural que afecta en mayor o menor medida a todos los Estados de la Unión Europea. En España, este problema se agudizará dentro de diez años con la jubilación de la generación conocida como el baby-boom lo que ha puesto en duda la viabilidad del sistema de reparto de la seguridad social diseñado en los años 1963 a 1966 cuando la situación social de España y las necesidades de protección eran muy distintas a las que imperan actualmente.

Además, con carácter coyuntural, desde el año 2006 España ha estado inmersa en una crisis económica como consecuencia de los efectos de la globalización económica en el ámbito financiero y por la caída del sector inmobiliario debida a una subida desproporcionada en los precios de este sector secundario. Este hecho ha provocado un descenso de cotizantes que ha suscitado que los partidos políticos se conciencien sobre la necesidad de que el sistema de reparto de la seguridad social se modifique para que sea sostenible en lo que respecta al pago de las pensiones públicas. A partir del año 2011, se han introducido reformas en relación a las pensiones

* Cómo citar / Citation 'Chicago-Deusto' (Autor-fecha / Author-date / Lista de referencias / Reference list entries): Gutiérrez Bengoechea, Miguel. 2018. «La tributación de las pensiones públicas». Estudios de Deusto 66, n. ${ }^{\circ}$ 1: 171-196. doi: http://dx.doi. org/10.18543/ed-66(1)-2018pp171-196. 
públicas; así, el retraso en la edad de jubilación, el envejecimiento activo, la regulación del factor de sostenibilidad, el índice de revalorización y una recomendación hacia los instrumentos de previsión social privados como complemento de las pensiones públicas pretenden asegurar el principio constitucional de la suficiencia económica de los ciudadanos.

\title{
Palabras clave
}

Pensión; envejecimiento; crisis; impuestos.

\begin{abstract}
The aging of the population is a demographic problem of a structural character which affects in a large or small measure all members of the European Union. In Spain, this problem will enlarge within the next 10 years with the retirement of former baby boom generation, which has put in doubt the viability of the social security economic distribution, designed in the years between 1963-1966, when the state of affairs of Spain and their protective needs were very distinct to the current existing ones.

Furthermore, with transitory character, Spain has been immersed in an economic crisis since 2006, due to the effects of the economic globalization in the financial field especially by the fall in the real estate sector caused by the disproportionate rise in the prices of this secondary sector. This fact has provoked a decline in contributors which has raised a necessary awareness in political parties that requires a sustainable modification in respect to public pension payments. From the year 2011 reforms have been introduced in relation to public pensions, so the delay in retirement age, the active aging, the regulation of the sustainability factor, the revaluation indicator and recommendation towards private social pension plans as a complement of public pensions, are aiming to secure the constitutional principle of economic sufficiency for citizens.
\end{abstract}

\section{Keywords}

Pension; Aging; crises; taxes. 


\begin{abstract}
SUMARIO: I. ASPECTOS GENERALES QUE INCIDEN NEGATIVAMENTE EN EL SISTEMA DE REPARTO DE LA SEGURIDAD SOCIAL. II. CRISIS DEL SISTEMA DE REPARTO DE LA SEGURIDAD SOCIAL EN ESPAÑA. III. REFORMAS EN EL SISTEMA DE REPARTO DE LA SEGURIDAD SOCIAL EN ESPAÑA. IV. SISTEMA DE REVALORIZACIÓN DE LAS PENSIONES PÚBLICAS. V. MEDIDAS FISCALES Y LABORALES PARA MEJORAR LA TASA DE DEPENDENCIA LABORAL Y DE SUSTITUCIÓN. ALGUNAS NOTAS SOBRE LA DESCENTRALIZACIÓN TERRITORIAL DE LA SEGURIDAD SOCIAL. VI. CONCLUSIONES.
\end{abstract}

\title{
I. ASPECTOS GENERALES QUE INCIDEN NEGATIVAMENTE EN EL SISTEMA DE REPARTO DE LA SEGURIDAD SOCIAL
}

La protección de la vejez ocupa un lugar medular en el seno del sistema de previsión social. De hecho, no sólo representa el capítulo más importante de las transferencias del Estado de Bienestar, sino que, además, se trata de la mayor y más importante política pública orientada a la vejez.

No obstante, hay que tener en cuenta que este protagonismo convierte a la financiación de esta contingencia en su modalidad contributiva en el principal foco de atención y la reciente crisis financiera y de la deuda soberana han contribuido a acelerar este proceso.

Actualmente todos los países que siguen un sistema de reparto de la seguridad social, que consiste fundamentalmente en que lo que se recauda por las cotizaciones de las personas en situación laboral activa se reparte entre las personas que están en una situación de inactividad laboral como consecuencia de la jubilación, están haciendo ajustes económicos para garantizar el pago de las pensiones.

Esta situación es propiciada de forma coyuntural por la crisis económicofinanciera de carácter mundial que todos los países de la eurozona están aún sufriendo y de forma estructural por la variación experimentada por el factor demográfico.

En el aspecto coyuntural, la recesión económica conlleva repercusiones en los mercados laborales, que lógicamente debilita el segmento de población en edad de cotizar. Además, las reformas laborales de los países de la Unión Europea tienden a flexibilizar el mercado laboral impulsando la contratación temporal y abaratando el despido del trabajador. En términos monetarios esta situación se traduce en salarios más bajos que inciden negativamente en el montante de las cotizaciones de los trabajadores y, con ello, en el sistema de financiación básico de las pensiones públicas.

A este panorama, no muy halagüeño, hay que añadir que estamos asistiendo en los países desarrollados a un envejecimiento exponencial ligado a la esperanza de la vida y a la disminución de la natalidad. 
En este sentido, en el año 1900 la esperanza de vida en España era aproximadamente de 35 años y en la actualidad es de 82 años y las previsiones del Instituto Nacional de Estadística es que la esperanza de vida para el año 2050 sea de 90 años. Además, en España para ese año se producirá un incremento de la población dependiente donde el 73 por ciento de los hombres y el 90 por ciento de las mujeres cumplirán los 80 años ${ }^{1}$.

Este mayor incremento en la esperanza de vida es causado por diversas circunstancias propias de un Estado de bienestar entre las que hay que destacar, la mayor calidad en la alimentación y a una sanidad cada vez más especializada $^{2}$.

Como contraposición, la tasa de natalidad europea ha descendido notablemente debido a factores que podemos clasificar en objetivos y subjetivos.

Los factores objetivos estarían conectados con el incremento del paro, la bajada salarial de los trabajadores de la eurozona y el hecho de la incorporación de la mujer al mercado laboral, todos estos factores inciden en que las mujeres retrasen la edad en la que deciden ser madres. En términos cuantitativos, los matrimonios que deciden tener hijos no llegan a tener por término medio más de dos.

En el aspecto subjetivo, se está dando el fenómeno de que las personas con cierto bienestar social, donde los dos cónyuges están trabajando, tienden a ser más egoísta desde un punto de vista patrimonial y son reticentes a perder calidad de vida en la crianza y educación de un hijo. Por otra parte, muchas personas no ven con buenas perspectivas el futuro laboral que podrían tener sus hijos, lo que les coarta en la decisión de ser padres.

\section{CRISIS DEL SISTEMA DE REPARTO DE LA SEGURIDAD SOCIAL EN ESPAÑA}

La situación descrita pone en entredicho el sistema de seguridad social diseñado en España en la Ley de Bases de la Seguridad Social de 1963 que consiste en que las aportaciones a la seguridad social de los trabajadores en activo se reparten entre la población jubilada. Este sistema exige una fuerte vinculación del pensionista con el trabajador a través de un contrato social entre generaciones por lo que es esencial un equilibrio entre población ocupada y jubilada que está igualmente ligado a la relación existente entre natalidad y mortalidad ${ }^{3}$.

1 Cfr. Fundación General. CSIC. Informe sobre el envejecimiento 2010: 20

2 El bienestar de las sociedades occidentales tiene mucho que ver con el envejecimiento como resultado de una reducción de la mortalidad y un alargamiento de la esperanza de vida. Cfr. Suarez Corujo 2014: 77.

3 El artículo 87 de la Ley de la Seguridad Social señala que el sistema de la seguridad social es el de reparto. 
En la década de los 60 y hasta 1975 llegó la generación del baby boom. En España nacieron 14 millones de niños, 4.5 millones más que en los 20 años siguientes y 2.5 millones más que en los 20 años anteriores. Con este hecho demográfico, el sistema se aseguraba su sostenibilidad porque bajo un sistema económico con un crecimiento estable ese aumento exponencial de la población ocuparía puestos de trabajo y las cotizaciones financiarían a la población jubilada. Además, la esperanza de vida era más corta porque no existía en la sanidad los avances tecnológicos como en los momentos actuales y la alimentación de calidad no estaba al alcance de toda la población.

Sin embargo, esta situación que inicialmente fue favorable para la economía española amenaza con poner en peligro el sostenimiento de las pensiones públicas. En primer lugar porque desde 1975 hasta el año 2004 el número de nacimientos empezó a descender hasta alcanzar 1.3 niños por mujer ${ }^{4}$. En segundo lugar, esta situación de desequilibrio demográfico como consecuencia de la futura jubilación de la generación del baby-boom incidirá coyunturalmente (al menos durante 15 años) en el pago de las pensiones. Al ser un problema temporal se exigen soluciones extraordinarias salvo que la situación económica en los próximos años dé un giro radical con un crecimiento del Producto Interior Bruto (PIB) en torno al 3 por ciento.

Debido a lo expuesto anteriormente, en la actualidad, en un Estado de Bienestar social en el que la calidad de vida ha mejorado notablemente para toda la población, se observa, una inversión de la pirámide poblacional, es decir, se está produciendo un ensanchamiento de la parte alta de la pirámide que se corresponde con la población jubilada y un estrechamiento en la base de la pirámide que se corresponde con la natalidad. Esta situación provoca que la tasa de dependencia configurada como el ratio de afiliados a la seguridad social dividido por los pensionistas esté disminuyendo de forma alarmante obligando al gobierno a compensar las desviaciones entre los ingresos y gastos de la seguridad social con el remanente que aún tiene la seguridad social española provenientes de años en los que había superávit por la alta tasa de empleo respecto al desempleo, por la menor esperanza de vida de la población española y por la entrada de la población inmigrante.

Sin embargo, las previsiones sobre los parámetros expuestos, no son favorables: en primer lugar para el año 2022 si el comportamiento demográfico sigue la misma tendencia, la tasa de dependencia será de un $58 \%$ lo que significa que de cada 10 personas trabajadoras habrá 6 que estarán en situación de desempleo y en 40 años la tasa de dependencia será del 100 por ciento, es decir, habrá un trabajador activo por cada trabajador inactivo.

\footnotetext{
4 Monereo Pérez y Fernandez Bernat 2014: 23 y ss.
} 
El problema del envejecimiento como expresa BORJA SUAREZ no sólo representa un desafío para el pago de las pensiones públicas sino que tiene impactos en el gasto público de otras partidas presupuestarias como por ejemplo la sanidad y las de dependencia fundamentalmente. Así, el gasto en sanidad se incrementaría en aproximadamente 1.3 puntos porcentuales del Producto Interior Bruto desde el 6.5 por ciento en 2010 al 7.8 por ciento en $2060^{5}$.

A esta situación habría que añadir la ineficacia del mercado laboral en España durante más de un lustro para absorber la demanda de empleo de jóvenes licenciados o graduados universitarios. Esta última situación provoca lo que se denomina fuga de talentos a países extranjeros, sobre todo, a Estados del norte de Europa generando un gran valor añadido en estos territorios, habiendo asumido España, el coste de su formación 6 . Pero lo más preocupante, es que se ha perdido una generación de potenciales cotizantes que dejará de financiar a otra que sí lo ha hecho a lo que habría que añadir como ya se ha expuesto la edad de jubilación de las primeras cohortes de la generación baby boom. Esta situación, provocará que el Estado tenga que asumir el incremento en el coste social de las pensiones durante un período de 15 años implicando un mayor esfuerzo presupuestario.

Otro aspecto, al que se ha hecho mención y que hay que destacar, es la inmigración que ha experimentado España, que ha pasado de representar el 1.6 por ciento de la población en 1998 al 12.2 por ciento de la población total en 2011.

El incremento desde el año 2000 de inmigrantes, sobre todo, en edad de trabajar y lejos de la jubilación, provocó que la estructura piramidal de la población española se fuese corrigiendo debido al incremento de la natalidad por parte de este sector de la población.

Por lo tanto, el efecto de la inmigración sobre las pensiones ha sido muy significativo porque esta población accede a puestos de trabajos que no sólo imprimirán riqueza al país de acogida sino que además, cotizan a la seguridad social contribuyendo a la cantidad que se repartirá entre la población inactiva laboralmente. Además, las remuneraciones que perciben estarán sometidas a tributación con lo que incrementarán la recaudación tributaria ${ }^{7}$.

No obstante, los efectos positivos hay que considerarlos en términos generales como coyunturales porque la población inmigrante que viene a un

5 Cfr. Borja Suarez 2014: 85.

6 La emigración del capital humano cualificado dificulta la recuperación económica porque impide la creación de empresas especializadas y con alta productividad. Cfr. Borja Suarez op. cit.: 77 .

7 A nivel europeo es posible conseguir con la inmigración una tasa de fecundidad de 2.1 niño por mujer para alcanzar la tasa deseada de reposición. Cfr. Ferreras Alonso 2010: 16 . 
determinado país, con el tiempo envejece y pasan a una situación de inactividad laboral y respecto a la mayor fecundidad que suelen tener inicialmente las inmigrantes tienden a converger en términos numéricos al del Estado de acogida.

En España este factor tuvo un efecto positivo en la época de crecimiento económico cuando la construcción estaba en auge y se necesitaba mucha mano de obra sin cualificar. Esta situación cambió a partir de la crisis económica en la que España aún está inmersa como consecuencia de una insuficiente demanda interna provocada por la caída del sector inmobiliario unida a la crisis internacional de carácter financiero, lo que ha provocado que el movimiento migratorio muestre un sentido negativo.

Todos estos aspectos redundan negativamente en los potenciales beneficiarios de las prestaciones públicas, pues posiblemente no pueden acceder en las mismas condiciones de igualdad. Esta circunstancia es ciertamente preocupante, en la medida que el modelo de reparto hace que los potenciales beneficiarios puedan estar contribuyendo al sistema al albur de unas falsas expectativas pues nadie puede asegurar que las condiciones de acceso a la prestación y la cuantía de las mismas sean modificadas en el futuro.

Hay que mencionar que en la actualidad, aunque la reforma laboral está empezando a crear empleo respecto a años en que la economía española estaba en plena crisis, este tipo de empleo es fundamentalmente temporal, a tiempo parcial y con bajos salarios, lo que redunda negativamente en la base de cotización de los trabajadores e indirectamente en las pensiones públicas.

Esta situación de precariedad laboral desemboca de nuevo en una desconfianza de las personas en realizar inversiones importantes como en la adquisición de una vivienda o lo que puede ser peor, retrasar la edad de ser padres o incluso anularla del proyecto de vida, impidiendo socialmente el relevo generacional en las pensiones públicas.

Para tener un conocimiento aproximado de las pensiones públicas que podrían percibir los trabajadores en el futuro habría que establecer un sistema de capitalización que no es el más adecuado dado que el importe del capital acumulado para la jubilación depende de la rentabilidad obtenida por los fondos privados donde se realizan las inversiones. La experiencia nos ha mostrado que el sistema de capitalización instaurado en algunos países sudamericanos, la cantidad a percibir depende del tipo de rentabilidad durante el tiempo en que se ha acumulado el capital. En este sentido, el sistema de capitalización, además de suponer una ruptura total con el esquema de solidaridad intergeneracional, no garantiza un tratamiento uniforme a los trabajadores, ya que los trabajadores pueden tener una prestación diferente en función de la rentabilidad de los fondos donde se han invertido sus cotizaciones sociales. Sin embargo, es cierto que desde una visión capitalista, estos instrumentos de ahorro favorecen la recuperación económica ya que el 
ahorro generado con estos instrumentos, normalmente, a través de las entidades financieras se reinvierte en la economía en forma de créditos y préstamos dirigidos a la creación de empresas e indirectamente a la creación de empleo.

Un ejemplo hacia este sistema de capitalización lo tenemos en Chile donde se produjo un cambio en su sistema de pensiones públicas pasando de un sistema de reparto al de capitalización parcial obligatoria y otra parte del coste de las pensiones es financiado por el Estado a través de impuestos. Esta situación produce un gap o vacío en la adaptación y convergencia entre dos sistemas tan dispares en el tratamiento de las pensiones públicas que lo hacen desaconsejable pues al menos una generación sufriría el ajuste.

Un segundo criterio que se utiliza en algunos Estados de la Unión Europea como Italia y Suecia es el sistema de cuentas nacionales.

Este sistema se basa en que el Estado abre una cuenta a cada trabajador donde se van acumulando todas las cotizaciones durante la vida laboral del trabajador. Una vez que éste alcance la edad de jubilación, la pensión que percibirá será el resultado de dividir el importe monetario del derecho consolidado en su cuenta nacional entre la esperanza de vida estimada de la cohorte de la población a la que pertenece el individuo.

Otros sistemas de seguridad social se basan en una combinación obligatoria del sistema de reparto con el de capitalización, como el caso de Paraguay y Argentina. En determinados países de Europa del Este como Hungría, Polonia, Letonia, Bulgaria, Croacia o Estonia combinan el sistema público de reparto con un régimen de cotizaciones definidas, gestionadas por entidades privadas o públicas. Sin embargo, Dinamarca, Alemania, Noruega, Finlandia y Suiza, financian una importante cantidad del coste de la pensiones a través de impuestos.

En Sudamérica, Estados como Bolivia, El Salvador y Nicaragua simplemente han sustituido el sistema público de reparto por el sistema de capitalización.

En España, aunque está establecido el sistema público de reparto, se utilizan los impuestos, para financiar las pensiones no contributivas y, se está pensando en financiar las pensiones de viudedad y orfandad con cargo a los ingresos públicos. No debemos olvidar que el artículo 31 de la CE dispone que:" Todos contribuirán al sostenimiento de los gastos públicos mediante un sistema tributario justo..." lo que legitima la utilización del tributo para financiar en su caso, las pensiones públicas.

Otra cuestión es que se utilice el tributo con carácter presupuestario o bien se introduzca un impuesto finalista, en mi opinión de carácter coyuntural para los posibles desajustes que se produzcan en el sostenimiento de las pensiones públicas.

Sin embargo, la utilización de impuestos para el pago de las pensiones tiene sus detractores argumentando que es mejor que exista una fuente 
monetaria afecta al pago de las pensiones y no una partida presupuestaria que depende sensiblemente de cómo esté el crecimiento económico de un determinado país.

\section{REFORMAS EN EL SISTEMA DE REPARTO DE LA SEGURIDAD SOCIAL EN ESPAÑA}

La reforma del sistema de la seguridad social surge con el Pacto de Toledo en el año 1995 formado por un conjunto de parlamentarios que se hicieron eco de las propuestas del Libro Blanco de la Unión Europea. Una de las propuestas más importante recogida en el Pacto de Toledo es la ampliación del número de años para el cálculo de la base reguladora que pasó de 8 a 15 años y además se incentivó la prolongación de la vida laboral.

A partir de las recomendaciones del Pacto de Toledo las políticas en torno a la seguridad social son menos expansivas ya que van condicionados por factores económicos y demográficos.

Fruto de los acuerdos entre sindicatos firmado el 9 de octubre de 1996 fue la promulgación de la Ley 24/1997, de 15 de julio, de Consolidación y Racionalización del sistema de Seguridad Social.

Esta Ley estaba dirigida a reformar el modelo de la seguridad social hacia la contención del gasto público.

En este sentido, se hizo una separación de las fuentes de financiación de la seguridad social, distinguiendo entre fuentes contributivas y no contributivas. En el aspecto teleológico, la norma lesiona el principio de solidaridad y se vislumbra a un Estado que empieza a demandar un carácter más asistencial ya que cada vez son mayores las necesidades de los ciudadanos y menor la respuesta del sistema público de la seguridad social. Todas estas medidas, por tanto, van dirigidas a sanear las cuentas del Estado y de esa forma restringir la acción protectora de la seguridad social.

Desde 1998 hasta el 2007 España registró una etapa de bonanza económica que fue esencial para constituir un fondo de reserva para el pago de las pensiones ya que el número de cotizantes se incrementó en 8000.000 de personas.

Sin embargo, la crisis inmobiliaria y financiera a nivel internacional desplegó en España sus efectos desde 2007 con una destrucción de empleo muy significativo lo que hizo que las autoridades se plantearan una reforma legislativa en prevención a la reducción del fondo de reserva para el pago de las pensiones y para el propio sostenimiento de las mismas a medio plazo.

La promulgación de la Ley 27/2011, de 1 de agosto sobre Actualización; Adecuación y Modernización del Sistema de la Seguridad Social pretendía la sostenibilidad del sistema de pensiones a corto y largo plazo tratando de reforzar la contributividad del sistema y manteniendo la proporcionalidad 
entre las contribuciones al sistema y las prestaciones esperadas del mismos. En esta norma se aprobaron medidas como: alargar la vida laboral de las personas hasta los 67 años, salvo para aquellos pensionistas con más de 38 años cotizados $\mathrm{y}$, asimismo, la consideración del número de años para el cálculo de la base reguladora pasó de 15 a 25 años $^{8}$.

El hecho de retrasar el acceso a la pensión de jubilación tiene el propósito de reequilibrar la relación entre trabajadores activos y jubilados. Así, la prolongación de la vida laboral tiene una incidencia dual en la base financiera de las pensiones públicas. En primer lugar, el incremento de la vida laboral incrementa las cotizaciones al sistema de la seguridad social y por otro lado, disminuye los años de percepción de las pensiones por lo que inciden negativamente en los gastos de las pensiones públicas.

Igualmente, el incremento del número de años que se utiliza para el cálculo de la base reguladora de la pensión pública disminuye el gasto en las pensiones porque normalmente en los últimos años de la actividad laboral es cuando los trabajadores suelen percibir mayores remuneraciones.

Sin embargo, esta situación puede jugar positivamente para aquellos trabajadores que como consecuencia de la crisis económica hubieran perdido sus puestos laborales en los años próximos a la jubilación?.

También se incrementan los incentivos para prolongar de forma voluntaria la vida laboral de aquellas personas que han alcanzado la edad legal de jubilación. Así, aquellas personas que decidan seguir desempeñando una actividad laboral una vez que tengan los años cotizados y la edad para jubilarse tendrán una prima en su base reguladora entre el 2 y el $4 \%$ por cada año trabajado sin que en ningún caso pueda superar la pensión máxima establecida legalmente aunque sí pueden aumentar su pensión por encima del 100 por ciento.

Todas las normas laborales aprobadas por el legislador español están en sintonía con el soft law de la UE en materia de las pensiones públicas. En este aspecto, el libro blanco de la Comisión Europea para la sostenibilidad de las pensiones públicas de 2012 establece unas recomendaciones para los

8 Aunque la Ley 21/2011, retrasó la edad de jubilación como refuerzo de la dimensión contributiva de la edad de jubilación. El artículo 161.1.a) Ley General de la Seguridad Social (RDley 8/2015, de 30 de octubre) mantiene la edad de jubilación a los 65 años siempre y cuando se hayan cotizado 38 años y 6 meses, en lugar de 35 que hasta entonces se exigían. En el caso de reunirse estos requisitos entonces la edad de jubilación se eleva a los 67 años y se consigue percibir la totalidad de la pensión si el tiempo cotizado llega hasta los 37 años.

9 Cfr. Monereo Pérez y Fernandez Bernet op.cit.: 37 y ss. Aunque también habría que valorar el tiempo que se toma como referencia para el cálculo de la base reguladora que como sabemos está establecido actualmente en 25 años. 
diferentes países de la Unión Europea. Para España se concreta en tres elementos básicos para la sostenibilidad:

a) Incremento de la edad de jubilación real.

b) Prolongación de la vida activa

c) Incremento de la participación en el mercado de trabajo

Estas propuestas se incorporaron a nuestro ordenamiento jurídico con la aprobación del RD-ley 5/2013, de 15 de marzo, de medidas para favorecer la continuidad de la vida laboral de los trabajadores de mayor edad y promover el envejecimiento activo que reguló la compatibilidad entre la percepción de la pensión de jubilación y el trabajo por cuenta propia o ajena para favorecer el alargamiento de la vida activa, reforzar la sostenibilidad del sistema de la seguridad social y aprovechar en mayor medida los conocimientos y experiencias de estos trabajadores. Esta posibilidad muy restringida en el ordenamiento español hasta la fecha, es habitual en las legislaciones de países del entorno. Se permite así que aquellos trabajadores que han accedido a la jubilación al alcanzar la edad legal y que cuentan con largas carreras de cotización puedan compatibilizar el empleo a tiempo completo o parcial con el cobro del 50 por ciento de la pensión, con unas obligaciones de cotización limitadas ${ }^{10}$.

A tenor de lo regulado en la norma se pretendía incidir fundamentalmente en la partida del gasto público para conseguir la sostenibilidad del sistema de las pensiones públicas. Además, se estableció que las pensiones contributivas se financiasen con las cotizaciones a la seguridad social y las no contributivas como las sanitarias y asistenciales a través de los Presupuestos Generales del Estado vía impuestos. No obstante, la norma no planteó la posibilidad de suprimir los límites que existen en la bases de cotización o en un aumento de los tipos de cotización ${ }^{11}$.

Durante el año 2013 fue promulgada sin contar con la opinión de los agentes sociales, la Ley 23/2013, de 23 de diciembre, reguladora del factor de sostenibilidad y del índice de revalorización del sistema de pensiones de la seguridad social, en el cual se introduce un factor de corrección conocido como factor de sostenibilidad desligándose además, la actualización de las pensiones del Índice de Precios al Consumo (en adelante IPC).

10 El artículo 2 del RD-ley 5/2013 establece que el trabajo compatible podrá hacerse a tiempo completo o parcial y una vez que finalice el cese de la actividad laboral se reestablecerá la percepción íntegra de la pensión de jubilación.

11 El fin de esta norma no es otro que atender al mandato que la Comisión Europea fijó en el Libro blanco de 2012 hacia la obtención de unas pensiones adecuadas, seguras y sostenibles. 
La norma define el factor de sostenibilidad como un instrumento cuyo fin es vincular el importe de las pensiones de jubilación del sistema de la seguridad social a la evolución de la esperanza de vida de los pensionistas de forma automática. De este modo se consigue que el importe total que perciba un pensionista que acceda al sistema dentro de ciertos años sea equivalente al que perciba el que se jubila en un momento anterior, para lo cual se relaciona la esperanza de vida estimada en ambos momentos.

Como manifiesta MONEREO FERNÁNDEZ y FERNANDEZ BERNAT el factor de sostenibilidad pretende que la pensión inicial de jubilación debe ajustarse de modo que el montante esperado de las pensiones que reciba a lo largo de su vida un pensionista que entre en el sistema en un momento determinado con una determinada base reguladora y una edad y, por tanto, con una esperanza de vida determinada, sea equivalente al que reciba un pensionista que entró en el sistema con la misma base reguladora y con la misma edad en un momento anterior y, por tanto, con una esperanza de vida inferior $^{12}$.

Sin embargo, la aplicación del factor de sostenibilidad sólo se aplica a las pensiones públicas una vez alcanzada la pensión de jubilación, pero no sería de aplicación a las pensiones que deriven de una situación de invalidez permanente, total, absoluta o gran invalidez ${ }^{13}$.

Con anterioridad a la regulación del factor de sostenibilidad, el sistema de reparto se instituía en la relación entre el trabajador y el pensionista. Con el factor de sostenibilidad mejora la equidad intergeneracional. A título ilustrativo, con el factor de sostenibilidad tanto el padre como el hijo percibirán las mismas pensiones públicas porque cada uno la percibirá en función de la esperanza de vida en el momento de la edad tomada para la jubilación; sin el factor de sostenibilidad basado en la esperanza de vida, el hijo gozaría de un mayor tiempo para disfrutar de las pensiones públicas al ser mayor la esperanza de vida cuando alcance La edad de jubilación.

En general el factor de sostenibilidad va a disminuir con carácter general las pensiones públicas porque la esperanza de vida normalmente va en crecimiento con el paso de los años. Aunque no siempre tiene que ser así, por ejemplo, si los servicios públicos no funcionan correctamente por motivos presupuestarios, sobre todo, los relacionados con la rama sanitaria podría haber un descenso en la esperanza de vida lo que invertiría el descenso de las pensiones públicas. Por otra parte, puede suceder como ya se ha reseñado con anterioridad que se incremente en los próximos años la productividad

12 Monereo Pérez y Fernandez Bernat op. cit.: 102.

13 En estos supuestos puede estar justificado la inaplicación del factor de sostenibilidad porque las lesiones complicarían la esperanza de vida para cada situación personal. $C f r$. Tortuelo Plaza 2014: 115. 
empresarial y el crecimiento económico y con ello las cotizaciones a la seguridad social paliando de esta manera el efecto negativo que tiene el factor de sostenibilidad en el pago del montante de las pensiones públicas. No obstante, hay que pensar que el sistema quiere demostrar que con un sistema de reparto de aportación definida, en vez de prestación definida, como hasta ahora, las pensiones pueden pagarse con este factor de sostenibilidad sin necesidad de acudir a otras fuentes de financiación.

Debemos pensar que el Estado se ha estado comprometiendo al pago de unas pensiones públicas durante toda la vida a las personas que cumplan con ciertos requisitos que van relacionados con los años cotizados y con la base de regulación a efectos de las cotizaciones. Sin embargo, a partir de 2019 con la introducción del factor de sostenibilidad el sistema será de aportación definida y la prestación dependerá de varios factores como: número de cotizantes, evolución económica del país y, sobre todo, de la esperanza de vida media de la población. No obstante, este factor tendrá que equilibrar la justicia social y el carácter redistributivo que según la Constitución Española tiene la seguridad social.

Una vez que se pueda corregir, al menos parcialmente, la tendencia de la tasa de dependencia, que garantizaría las pensiones públicas, habría que incidir en lo que se denomina tasa de sustitución, es decir, el poder adquisitivo que pierde el trabajador cuando pasa al retiro laboral. Este concepto es importante porque está regulado en términos generales en la Constitución Española (en adelante CE), en concreto el artículo $50 \mathrm{CE}$ dispone que: «Los poderes públicos garantizarán mediantes pensiones adecuadas y periódicamente actualizadas, la suficiencia económica a los ciudadanos durante la tercera edad». A tenor de este precepto, el Estado debe realizar políticas de gasto público e incentivar a los ciudadanos a la contratación de instrumentos de previsión social que completen las pensiones públicas con el objetivo de que el bienestar social permanezca estable.

Sin embargo, el problema no está en la restricción presupuestaria porque el gasto público en pensiones en España, está por debajo de la media de la Unión Europea. El problema está centrado en el ingreso público para financiar las pensiones públicas.

Actualmente la seguridad social satisface las pensiones públicas de carácter contributivo con las cotizaciones a la seguridad social; además de las pensiones de viudedad y orfandad. Sin embargo, las prestaciones asistenciales, las pensiones no contributivas y para de las contributivas que no llegan al mínimo para cobrar la pensión (las llamadas complementos por mínimos) son financiadas por el Estado con cargo a los Presupuestos Generales del Estado.

En este aspecto, el Gobierno tendría que plantearse el desvincular la financiación de las pensiones de viudedad y las de orfandad con las 
cotizaciones obligatorias a la seguridad social y financiar estas partidas con cargo a los Presupuestos Generales del Estado vía impuestos y, en su caso, aumentar las cotizaciones a la seguridad social para las rentas del trabajo más altas.

Hay países como Dinamarca que van más allá, al desvincular el pago de las pensiones de las cotizaciones del trabajador. Este país define el Derecho a percibir una pensión de vejez como un Derecho de ciudadanía no vinculado a la vida laboral previa de la persona y financiar todas las prestaciones con la recaudación obtenida por impuestos.

Ante esta situación, cabría una medida de dudosa legitimidad que conllevaría bajar las pensiones públicas con carácter coyuntural, al menos hasta que desaparezca el efecto del baby-boom, manteniendo un mínimo vital que satisfaga las necesidades básicas de las personas jubiladas. Esta situación no sería justa desde el punto de vista de la equidad ni de la justicia social. No obstante, esta situación tendría sentido si estuviéramos ante un escenario de descentralización territorial de las pensiones públicas en la que el Estado mediante un fondo específico de compensación interterritorial compensara a las Comunidades Autónomas con menor renta per-cápita. Esta situación comportaría la cesión de más competencias tributarias a los entes autonómicos y establecer unos adecuados puntos de conexión en términos de residencia laboral para que no se planteen situaciones de conflictos en la aplicación de la norma tributaria.

\section{SISTEMA DE REVALORIZACIÓN DE LAS PENSIONES PÚBLICAS}

El libro blanco (2012) sobre «Agencia para unas pensiones adecuadas seguras y sostenibles» aduce que pequeñas oscilaciones en la cuantía de las pensiones públicas más bajas puede dar lugar a tasas de pobreza muy significativas en cierto sector de los pensionistas. Es esencial que las entidades públicas tomen conciencia de este hecho y prevengan que las tasas de reemplazo sean elevadas.

Para que no se produzca este empobrecimiento de la población jubilada es necesario que las pensiones sean dignas y se vayan revalorizando con arreglo a un índice que recoja el incremento del nivel de vida.

De esta forma, se cumpliría con el mandato constitucional regulado en el artículo 50 de la CE el cual obliga a los poderes públicos a garantizar la suficiencia económica durante la tercera edad mediante pensiones adecuadas y periódicamente actualizadas, aunque siempre han estado revalorizándose acorde al IPC desde el Informe de Evaluación y Reforma del Pacto de Toledo de 2011 se barajaron otros criterios sobre la actualización de las pensiones públicas que tomaron en consideración otros índices como el crecimiento de 
los salarios, la evolución de la economía o el cómputo de la totalidad de las cotizaciones a la seguridad social al igual que otros países de nuestro entorno.

Hacer depender las pensiones públicas del crecimiento de la economía supone que en épocas de recesión económica las revalorizaciones de las pensiones públicas son inferiores al crecimiento salarial sin que aquellas tengan que sufrir una revalorización negativa ${ }^{14}$.

La crisis económica que España sufre desde 2007 ha incidido negativamente sobre la revalorización de las pensiones públicas. En el año 2011, por ejemplo, se suspendió la revalorización de las pensiones públicas excepto para las pensiones no contributivas y las mínimas.

Con la promulgación de la Ley 23/2013 y la limitación sobre el endeudamiento público contenido en el artículo 135 de la CE se regula un nuevo sistema para la revalorización de las pensiones públicas ${ }^{15}$.

En concreto, el artículo 7 Ley 23/2013 modificó el artículo 48 del Texto refundido de la Ley General de la Seguridad Social de modo que las pensiones públicas serán incrementadas en función de un índice de revalorización previsto en la Ley de Presupuestos Generales del Estado que va a estar ligado a variables de tipo presupuestario como el crecimiento de los ingresos, el número de pensiones; así como por los efectos de sustitución entre pensionistas ${ }^{16}$.

Este índice de revalorización no admite signo negativo, ni tan siquiera que el índice fuese nulo, aun cuando se produzca una disminución en el crecimiento económico. Se prevé en la norma social que las pensiones públicas de las seguridad social y las demás correspondientes a las clases pasivas tendrán como mínimo una revalorización del 0.25 por ciento pero por otra parte, no podrán ser superior a la variación porcentual del índice de precios de consumo en el período anual anterior a diciembre del año t, más un 0.5 por ciento.

El hecho de revalorizar las pensiones tiene un trasfondo económico y social muy importante porque como se ha reseñado una gran parte de la población española está en situación de retiro laboral y podrían empobrecerse con las repercusiones sociales que ello conlleva en un estado de bienestar como el de España. Pero además del empobrecimiento que podría originarse para un amplio espectro de la población española no lo es menos para casi 4,5 millones de hogares que dependen exclusivamente de las pensiones de los familiares con los que en muchos casos conviven.

\footnotetext{
14 De La Puente Lavín 2006: 187.

15 El artículo 135 CE dispone que:” Todas las administraciones públicas adecuarán sus actuaciones al principio de estabilidad presupuestaria". Del precepto transcrito es evidente que la seguridad social está sometida al principio de estabilidad presupuestaria lo que se traduce en que debe vigilar la posibilidad de incurrir en un déficit que supere los márgenes establecidos por la Unión Europea para sus Estados Miembros.
}

16 Monereo Pérez y Fernández Bernat op. cit.: 120. 
Esta situación analizada desde un punto de vista económico crea un círculo cerrado de estancamiento del consumo privado porque ante una insuficiente revalorización de las pensiones públicas, puede haber un descenso en el consumo de esta importante parte de la población española con el impacto negativo que conllevaría en la creación y mantenimiento de las empresas y con ello en la creación de empleo.

En concordancia con la revalorización de las pensiones públicas, el Tribunal Constitucional en la STC 134/1987, de 21 de julio afirma que de la lectura de los artículos 41 y 50 de la CE, el Estado no está obligado a mantener las pensiones previstas inicialmente. Esta cuestión se traduce en que los afiliados a la seguridad social no ostentan un derecho subjetivo a una determinada pensión cuando alcancen la edad de jubilación o lo que es lo mismo, las prestaciones de la seguridad social no tienen por qué ser proporcionales a las cotizaciones y contribuciones de los afiliados.

No obstante, para cumplir con el principio de suficiencia en las pensiones públicas, las prestaciones no deben ser mínimas, sino que tendrían que ser suficientes y adecuadas, es decir, deben permitir un nivel de vida no muy inferior al que mantenían mientras estaban en una situación de actividad laboral activa.

La STC 190/1990, de 30 de mayo incide en que no se deben congelar las pensiones públicas de forma ilimitada en el tiempo y si es de forma temporal habría que actualizar en todo caso las pensiones más bajas y mínimas ya que en caso contrario no se garantizaría el poder adquisitivo de aquellos pensionistas cuyo único ingreso es la pensión pública.

Desde un punto de vista jurídico aunque el cambio en el sistema de revalorización de las pensiones no está afectado por la retroactividad en el sentido de que este cambio no afecta al concepto de pensión pública sino únicamente al sistema de cálculo de las mismas. Sí podría incidir negativamente en la confianza que los ciudadanos tienen en la estabilidad de sus derechos cuando éstos son modificados de forma desproporcionada ${ }^{17}$.

\section{MEDIDAS FISCALES Y LABORALES PARA MEJORAR LA TASA DE DEPENDENCIA LABORAL Y DE SUSTITUCIÓN: ESPECIAL REFERENCIA A LOS PLANES DE PENSIONES}

Las medidas o recomendaciones que se van a exponer a continuación sin duda van a incidir positivamente en la sostenibilidad de las pensiones

17 Vid. STC 234/1990. En definitiva, podría suscitarse una lesión al principio de seguridad jurídica en las expectativas de los ciudadanos en la estabilidad normativa que incide directamente en una cuestión tan trascendente como las remuneraciones de los pensionistas. Cfr. Juarez Corujo 2014: 40. 
públicas, sin embargo, lo difícil no es proponer medidas económicas, laborales o fiscales sino en qué medida aplicar cada una de ellas, es decir, cuáles hay que aplicar, cuándo y en qué medida.

Inicialmente, dado que el factor de sostenibilidad basado en la esperanza de vida de las personas físicas reducirá las pensiones públicas contributivas, una de las propuestas, va dirigida a incentivar que las personas físicas contraten un plan de previsión social alternativo como complemento a la pensión pública del Estado.

Aunque hay varios instrumentos de previsión social privados como los planes de pensiones, los planes de previsión asegurados y los planes de previsión empresarial, nos centraremos en los planes de pensiones ya que son los más contratados pues son ofertados mayoritariamente por las entidades financieras mientras que los otros dos sólo pueden ser ofertados por las entidades aseguradoras.

Este plan de previsión social de carácter privado permitirá que las personas físicas cuando se jubilen puedan complementar lo percibido por la pensión pública minimizando en gran parte la tasa de sustitución y así, garantizar casi el mismo poder adquisitivo que tenía el trabajador antes de jubilarse. Desde la regulación de los planes de pensiones por la Ley 8/1987, de 8 de junio, estos instrumentos de previsión social siempre han estado bonificados fiscalmente aun cuando a finales de los años 80 no existían los problemas que presentan actualmente el pago de las pensiones públicas.

No obstante, hay que hacer algunas matizaciones respecto a los planes de pensiones. En principio estos instrumentos de previsión privados generan rentabilidades financieras superiores a los sistemas públicos aunque es verdad que no se puede asegurar a los ahorradores los riesgos inherentes a esta inversión como por ejemplo la inflación, tipos de interés, índices bursátiles y una posible quiebra del sistema financiero aunque, en determinados casos, las entidades financieras garantizan la titularidad de las cantidades aportadas cuando se produce el rescate de los planes de pensiones.

Esta situación no se produce con los sistemas de reparto de la seguridad social porque éstos se mantienen más estables durante más tiempo en su capacidad de actuación como estabilizadores automáticos lo que sirve para reducir las necesidades sociales de la ciudadanía agravadas en esa coyuntura y al mismo tiempo para sostener el consumo y así evitar una caída más intensa de la actividad económica.

Analizando fiscalmente los sistemas privados de capitalización, en concreto los planes de pensiones, la Ley 35/2006 del Impuesto sobre la Renta de las Personas Físicas (en adelante IRPF) regula una serie de reducciones en la base imponible general de este impuesto que reducen sensiblemente el gravamen de este impuesto a aquellas personas que cumplan unos requisitos ligados a la actividad desarrollada, a la cuantía de las aportaciones y en 
situaciones especiales, de incapacidad o dependencia, se tienen en cuenta los aspectos subjetivos de los contribuyentes.

El artículo 51 LIRPF regula la posibilidad de que los contribuyentes opten por reducir la base imponible general del IRPF las aportaciones realizadas por los partícipes a planes de pensiones, incluyendo las contribuciones del promotor que les hubiesen sido imputadas en concepto de rendimiento de trabajo.

Sin embargo, este beneficio fiscal tiene un límite tributario regulado en el artículo 52 LIRPF para el conjunto de instrumentos de previsión social privados.

El precepto marca como cantidad máxima a reducir la menor de dos cantidades:

a) El 30 por ciento de la suma de los rendimientos netos del trabajo personal y de actividades económicas percibidas individualmente en el ejercicio.

b) 8000 euros anuales.

Habría que hacer algunas matizaciones sobre la contratación de este instrumento de previsión privada que se dirige fundamentalmente a complementar el sistema público de las pensiones públicas.

En primer lugar, el artículo 52 LIRPF discrimina en la aplicación de este beneficio fiscal a parte de la población cuya actividad sea el arrendamiento de bienes inmuebles o la inversión en valores mobiliarios o simplemente que se dediquen a la especulación enajenando y comprando bienes y derechos ${ }^{18}$.

Las personas físicas empiezan a tener ciertos recelos a estos instrumentos de previsión, regulados fundamentalmente en el Impuesto sobre la Renta de las Personas Físicas, ya que la fiscalidad de los mismos, sobre todo, las condiciones de acceso a los beneficios fiscales están continuamente modificándose y ello no permite al contribuyente tener una seguridad jurídica en cuanto a su planificación fiscal. Además, hay que considerar la rigidez en cuanto a su rescate que presentan estos instrumentos de previsión privada, lo que impiden a las personas físicas que contrataron un plan de pensiones movilizar sus derechos consolidados a otros instrumentos de ahorro privado que les pueda generar mayor rentabilidad ${ }^{19}$.

18 Este sector de la población se ve compelido a diseñar la financiación de su jubilación con aportaciones a seguros de vida o a cualquier otro instrumento financiero que les reporte una renta vitalicia acorde a su estándar de vida.

19 Se ha introducido la posibilidad de rescatar las aportaciones realizadas con más de 10 años de antigüedad. En concreto la Ley 26/2014 de reforma fiscal introdujo una nueva ventana de liquidez para los productos de ahorro inversión. En esta norma se regula el 
En colación a lo anterior, el legislador ha derogado recientemente, parte del beneficio fiscal en las aportaciones a los planes de pensiones para los contribuyentes con más de 50 años. Este cambio normativo, no tiene mucho sentido para los planes de pensiones que están asociados al ciclo vital de las personas físicas. Las personas físicas a partir de los 50 años empiezan a tener menos cargas familiares y la mayoría sin cargas hipotecarias. Esta situación les permite tener más liquidez y pueden aportar más cantidades a los planes de pensiones. Este colectivo es el que en un futuro no muy lejano va a experimentar el pago de las pensiones públicas acorde a unas estimaciones sobre la esperanza de vida por lo que posiblemente, busquen otros instrumentos de ahorro para completar sus pensiones públicas.

Otra de las objeciones que se pueden hacer a los planes de pensiones, es que al ser un instrumento que busca el que las personas físicas coloquen parte de sus ahorros, no son equitativos y se contraponen a la naturaleza redistributiva del Impuesto sobre la Renta de las Personas Físicas, en el sentido de que sólo las personas con mayor capacidad contributiva tienen la posibilidad de invertir parte sus ahorros en estos instrumentos de previsión privada que son en definitiva los que no van a sufrir el descenso en sus pensiones públicas.

Esta situación va a empobrecer a las personas con rentas más bajas cuyas pensiones se verán disminuidas por tener una base reguladora más baja y por la imposibilidad de completar las pensiones públicas con un sistema de capitalización privado.

Otra modificación que el legislador realizó en los instrumentos de previsión social es la eliminación de la reducción del $40 \%$ en las percepciones de los planes de pensiones, lo que condiciona a los partícipes a rescatar la percepción del plan de pensiones a través de una renta vitalicia y a evitar parcialmente la progresividad del IRPF.

Respecto a la consideración jurídica de las rentas percibidas por los instrumentos de previsión privados, habría que estudiar la posibilidad de que sólo tributara el rendimiento generado, es decir, la diferencia entre ingreso y gastos y catalogados jurídicamente como capital mobiliario. Al ser considerado como instrumento de ahorro tendría que integrarse en la base imponible del ahorro en el IRPF sometido a unos tipos impositivos fijos en función del rendimiento generado.

Desde una visión presupuestaria, habría que desvincular las pensiones de viudedad y las de orfandad de los ingresos de las cotizaciones sociales y financiarlas a través de impuestos.

derecho de los partícipes a rescatar las aportaciones con más de 10 años de antigüedad contando a partir del 1 de enero de 2015 la cuales se percibirán a partir del 1 de enero de 2025. Queda pendiente de desarrollo reglamentario los límites cuantitativos del rescate de las aportaciones realizadas. 
Esta sería una medida coyuntural tendente a financiar las jubilaciones de la generación del baby boom, las cuales, incrementarán el coste de la seguridad social considerablemente. Para entonces se espera que el crecimiento económico se haya relanzado y pueda atenuar el coste de las pensiones debido al incremento de los pensionistas.

Insistiendo en la misma figura impositiva, en mi opinión, se podría establecer un sobre gravamen finalista en el IRPF a través de la escala de gravamen progresiva que se aplica a la base imponible general del IRPF dirigida al pago de las pensiones. Sin embargo, el gravamen finalista tendría que articularse jurídicamente de manera que los contribuyentes que tendrían que contribuir, serían aquellos cuya base imponible general del IRPF superase la pensión máxima anual que fija la seguridad social para que no se produjese detracciones de algunos pensionistas para pagar a otros pensionistas.

Desde la imposición indirecta, en mi opinión, habría que incrementar los tipos impositivos de los impuestos especiales como: alcohol y tabaco fundamentalmente, sobre todo, por el incremento del coste sanitario que conllevan para la población en general pero con más intensidad en la población jubilada.

Si el problema se tornase de carácter estructural y, por tanto, las medias coyunturales no tuvieran el efecto requerido, entonces habría que tratar el coste de las pensiones públicas, como ya se ha apuntado, desde un punto de vista presupuestario; es decir, en el montante de los gastos públicos estaría incluido el coste de las pensiones tanto contributivas como no contributivas, siendo ambas financiadas por los ingresos públicos, entre los que estarían lógicamente además de los tributos, todas las cotizaciones a la seguridad social, pero sin estar afectos estos ingresos públicos al pago de las pensiones públicas.

En este escenario, el Estado puede tener un mayor control en el sostenimiento de las pensiones públicas porque podría incrementar algunas de las partidas de los ingresos públicos, bien mediante la elevación de los tipos de gravamen de algunas figuras tributarias como, por ejemplo, las relacionadas con la imposición indirecta o bien simplemente mediante la reducción de otras partidas presupuestarias relacionadas con el gasto público pero siempre respetando el principio de estabilidad presupuestaria.

A título ilustrativo, y si perjuicio de lo expuesto anteriormente el legislador a iniciativa del gobierno podría plantearse las siguientes medidas específicas de carácter tributario.

1. Reestablecer la deducción en el Impuesto sobre Sociedades de la deducción por aportación a planes de pensiones de los trabajadores ${ }^{20}$.

$20 \quad$ El artículo 43 del RDLeg 4/2004, de 5 de marzo (Texto Refundido del Impuesto sobre Sociedades) disponía que:'El sujeto pasivo podrá practicar una deducción en la 
2. En el Impuesto sobre la Renta de las Personas Físicas se deberían ampliar los límites de las aportaciones a los planes de pensiones, diferenciando las cantidades objeto de reducción en función de la edad del contribuyente y de los años que hubiese cotizado a la seguridad social.

3. Se tendría que volver a hacer una diferenciación entre las aportaciones del promotor y las del trabajador en relación a los límites de reducción aplicables a las aportaciones a los planes de pensiones objeto de reducción en la base imponible general del Impuesto sobre la Renta de las Personas Físicas. Sin embargo, en este aspecto como el beneficio fiscal de los planes de pensiones muestra un carácter regresivo, tan sólo tendría que estar bonificada, en mi opinión, la aportación del promotor a contribuyentes de rentas salariales medias y bajas, negando la posibilidad de reducir la base imponible general a las aportaciones hechas por el promotor a los contribuyentes con rentas más altas ${ }^{21}$.

4. Respecto a la consideración jurídica de las rentas percibidas por los instrumentos de previsión privados, habría que estudiar la posibilidad de que sólo tributase el rendimiento generado, es decir, la diferencia entre ingresos y gastos habría que calificarlo como rendimiento de capital mobiliario, y éste, como procede de un instrumento de capitalización, tendría que integrarse en la base imponible del ahorro sometido a unos tipos impositivos fijos en función de los rendimientos generados ${ }^{22}$.

5. Están surgiendo determinados seguros privados que establecen la exención tributaria del importe total percibido como ocurre con los seguros individuales de vida a largo plazo los cuales están diseñados para que la persona física ahorre y complemente la pensión pública. Tan sólo se exige que se mantenga el plan de ahorro durante cinco años y se aporte una cantidad anual inferior a 5000 euros.

6. También en el propio IRPF se debería estudiar la posibilidad de incrementar el beneficio fiscal cuando el contribuyente tuviese cargas familiares de manera que aquellos matrimonios que tuviesen 3 o más hijos, el legislador tendría que incrementar notablemente las deducciones fiscales familiares. Con esta propuesta, se podría incentivar en alguna proporción la natalidad.

7. En el Impuesto sobre Sucesiones y Donaciones se tendría que estudiar la conveniencia de aumentar la reducción actual que existe en

cuota íntegra del 10 por ciento de las contribuciones empresariales imputadas a favor de los trabajadores con retribuciones brutas anuales inferiores a 27.000 euros siempre que tales contribuciones se realicen a planes de pensiones de empleo".

${ }^{21}$ Cfr. Sanchez Pedroche 2006: 58

22 Cfr. Arranz De Andrés 2010: 61. 
las distintas normativas autonómicas e intentar igualarlas en su cuantía, en relación con la herencia de los seguros de vida como consecuencia del fallecimiento del tomador del seguro cuando éste no coincide con el beneficiario del mismo. No obstante, en mi opinión lo ideal sería asimilar el tratamiento fiscal que tienen los planes de pensiones en el Impuesto sobre Sucesiones y Donaciones al que tienen los seguros de vida, es decir, dejar fiscalmente la operación no sujeta a dicho impuesto 23 .

Entre las medias específicas que podrían adoptarse en el campo laboral para contribuir a la recuperación económica y con ello para recaudar más ingresos públicos para el pago de las pensiones públicas podríamos señalar las siguientes:

1. El legislador podría bonificar temporalmente las cotizaciones que las empresas tienen que realizar por sus trabajadores diferenciando según la edad de los trabajadores, lo que supondría inicialmente un mayor coste financiero para la Administración pero con el tiempo puede redundar en la creación de empleo por las empresas y con ello aumentar la masa de cotizantes a la seguridad social.

2. En colación con el punto anterior se podría plantear el destopar la base de cotización de los trabajadores. Esta medida no sólo originaría mayores ingresos públicos afectos al pago de las pensiones contributivas sino también evitaría una ruptura del principio de contributividad y de progresividad, pues las rentas más altas quedan exoneradas de cotizar por las cuantías que sobrepasan dicho tope, máxime cuando en los últimos años ni siquiera han evolucionado conforme el IPC real. Se estima que un incremento progresivo en el tiempo, hasta alcanzar, al menos, un 15 por ciento de la base máxima supondría una mejora del 0.18 por ciento del PIB $^{24}$.

3. Retrasar la edad de jubilación en función de la esperanza de vida de la población española. En este sentido, no siempre tiene que ir aumentando la esperanza de vida al mismo ritmo, es más, se podría producir una ralentización en el incremento de la esperanza de vida, si persisten los efectos de la crisis económica y determinados servicios públicos pierden calidad o lo que es peor que disminuya la prestación de los mismos por falta de una adecuada dotación presupuestaria.

23 En muchas ocasiones los planes de pensiones diferencian entre el tomador y el beneficiario, siendo personas físicas distintas lo que coincide con los aspectos subjetivos regulados en los seguros de vida.

${ }^{24}$ Cfr. Monereo Pérez y Fernandez Bernat op. cit.: 167 
4. El Estado podría añadir legalmente un sistema de capitalización en la seguridad social de carácter complementario, es decir, combinar el sistema de reparto con el de capitalización de manera que mediante Ley se obligara a las empresas a detraer de los salarios del trabajador un porcentaje para ingresarlo en un plan de pensiones de empleo de carácter público aunque podrían ser gestionados por entidades financieras. De esta forma, la pensión pública que le pueda quedar al trabajador una vez alcance la edad de jubilación podría ser complementada con el rescate de un instrumento de previsión social. Esta situación, sería considerada por los ciudadanos como una prestación pública de carácter coactivo establecida por el ente público y que bajo el artículo 31.3 de la Constitución tendrá que ser establecida con arreglo a la Ley.

5. Respecto a los profesionales que ejerzan la actividad como autónomos, las cotizaciones a la seguridad social tendrían que estar en relación a las rentas reales y no, a cantidades fijas predeterminadas legalmente

6. Algunas notas sobre la descentralización territorial de la seguridad social.

Cabría también la posibilidad de descentralizar territorialmente el sistema de la seguridad social. Esta situación no es que mejore el problema del sostenimiento de las pensiones públicas, pero sí, descargaría al Estado de la previsión y gestión de las pensiones públicas transfiriendo a las Comunidades Autónomas (en adelante CCAA) las competencias para el pago de las pensiones, sin perjuicio, de que el Estado tuviera que transferir fondos para compensar las diferencias en el nivel de las prestaciones y hacer efectivo el principio de solidaridad interregional.

Este es un tema complicado porque el Estado tiene competencia exclusiva sobre la seguridad social tal como lo recoge el artículo 149.1.17 de la CE el cual dispone que corresponde al Estado la:"Legislación básica y régimen económico de la Seguridad Social, sin perjuicio de la ejecución de sus servicios por las Comunidades autónomas".

En este sentido, si el Estado descentralizase el pago de las pensiones públicas en las CCAA podrían darse algunos problemas relacionados con la justicia redistributiva en el montante de las pensiones percibidas por los residentes en determinadas CCAA a lo que habría que añadir el fuerte coste de gestión que tendrían que asumir las CCAA.

Además, habría severas desigualdades entre las distintas CCAA en atención a la población activa que resida en cada autonomía ya que las CCAA con menos población activa, sus residentes tendrían menores pensiones públicas que los pensionistas residentes en CCAA con mayor riqueza, y, por tanto, con mayor empleo. 
No obstante, no todas las situaciones serían desfavorables para los residentes en situación laboral activa en CCAA con menor PIB per cápita: a título ilustrativo, en el caso de los funcionarios de cuerpos estatales percibirán las mismas rentas con independencia de la CCAA en la que residirían, lo que se traduciría, en que aquellos funcionarios que desarrollen sus funciones públicas en CCAA más pobres tendrán un mayor poder adquisitivo respecto los trabajadores públicos que residen en CCAA más ricas y por tanto con un nivel de precios más alto, lo que les permitiría incrementar su capacidad de ahorro respecto de otros compañeros de profesión que residan en CCAA con mayor nivel de vida. Esta situación de mayor capacidad de ahorro durante su vida activa, que también podría trasladarse a los trabajadores de empresas privadas que tengan sus retribuciones anuales muy altas, les permite compensar durante el tiempo de inactividad laboral como consecuencia de la jubilación la pérdida de ingresos incluso presentar una situación de superávit si comparamos el total de su ciclo vital en relación a la esperanza media de vida.

En esta hipotética situación de descentralización territorial de las pensiones públicas lo más complicado sería establecer los puntos de conexión, es decir, en base a qué criterios territoriales ligados a una actividad laboral, una CCAA está legalmente obligada a satisfacer la pensión pública a una determinada persona física.

\section{CONCLUSIONES}

El envejecimiento de la población es un problema de primera magnitud que como tal exige una respuesta global de los poderes públicos por la implicación económica y social que tiene. De no tomar acciones públicas para atenuar el problema descrito, el modo de producción de riqueza y el tipo de sociedad en que vivimos estarían abocados a la quiebra.

España al igual que muchos países de la Unión Europea están llevado a cabo modificaciones de gran calado en el sistema de la seguridad social confluyendo todas en la regulación de un factor de sostenibilidad que parte de la base de que el sistema de prestación definida debe sustituirse por el sistema de aportación definida en el que una vez alcanzada la edad de jubilación lo que se percibirá estará en función de la esperanza de vida de la población tomando como referencia un determinado año.

Sin duda, con este sistema de percepciones públicas basadas en el factor de sostenibilidad van a disminuir la cuantía de las pensiones públicas percibidas porque la misma prestación se va a repartir durante más años.

La Ley 23/2103 donde se regula de forma efectiva el factor de sostenibilidad no distingue el cálculo de la esperanza de vida en función del 
sexo ni del tipo de actividad laboral que ha desarrollado la persona que accede a la jubilación, a pesar de haber diferencias significativas. De lege ferenda, el legislador tendría que tomar en cuenta estas diferencias para que el sistema de reparto de la seguridad social ganase en justicia y equidad social.

Por otra parte, al desvincular la actualización de las pensiones públicas del incremento del nivel de vida, es decir, del IPC se corre el riesgo de empobrecer a un sector de la población que perciba las pensiones contributivas más bajas. En mi opinión, el Estado aunque apuesta por la reducción del gasto público para dar viabilidad al sistema de reparto de la seguridad social debería plantearse dar un mayor protagonismo a los ingresos tributarios en el pago de las pensiones públicas asumiendo la financiación de las pensiones de viudedad y las de orfandad, al menos, hasta que España llegue a una senda de crecimiento económico cercano al 3 por ciento del Producto Interior Bruto y pase el incremento del gasto público que durante 15 años se producirá con la jubilación del baby-boom.

No obstante, a pesar de que el concepto de envejecimiento de la población parece indicar sólo consecuencias negativas desde un punto de vista social y económico, hay también aspectos positivos que habría que destacar: en primer lugar el incremento en el envejecimiento de la población es un indicador del estado de bienestar de un país que confluye en la eficiencia y eficacia con la gestión y funcionamiento de los servicios públicos.

En el plano económico, el envejecimiento poblacional a nivel europeo toma como destino para disfrutar de la tercera edad a España, por muchos factores como el clima, cultura, dieta mediterránea, etcétera. Este hecho se traduce en que ciudadanos extranjeros, sobre todo, europeos elijan España como residencia para disfrutar de la vejez, realizando fuertes inversiones en capital inmobiliario bien, adquiriendo propiedades o alquilando estancias en residencias españolas enfocadas para el tercer ciclo de la vida. En ambos casos la incidencia en el PIB es positiva.

\section{BIBLIOGRAFÍA}

Arranz De Andrés, C. Fiscalidad de los planes de pensiones transnacionales y transfronterizos en el IRPF e IRNR, Wolkers Kluwer- CISS (Valencia, 2010): 61.

BorJa Suarez, C. El sistema público de pensiones: Críticas, Reformas y Sostenibilidad. Lex-Nova (2014): 85.

De La Puente Lavín, M. El sistema de pensiones en España. Evolución y perspectiva de futuro, Comares, (Granada, 2006): 187.

Ferreras Alonso, F. «Cambio demográfico y pensiones de la seguridad social» Fundación Alternativas. Documento de trabajo, n. ${ }^{\circ}$ 161, (2010): 16.

FundACIÓN GENERAL. CSIC. Informe sobre el envejecimiento, (Madrid, 2010): 20. 
JuARez Corujo, B. «Las sombras del inquietante proyecto de la Ley reguladora del factor de sostenibilidad y del índice de revalorizaciones del sistema de seguridad social», Documentación Laboral, n. ${ }^{\circ}$ 99, (2014) 40.

Monereo Pérez, JM. y Fernandez Bernat, JM. La sostenibilidad de las pensiones públicas, Tecnos (2014).

Sanchez Pedroche, JA. Ahorro y Fiscalidad, Edersa, (Madrid, 2006): 58.

SuArez Corujo, B. El sistema público de pensiones: Crisis, reforma y sostenibilidad, Lex-Nova, (2014): 23 y ss.

Tortuelo PlazA, JL. «Ley 23/2013, de 23 de diciembre, reguladora del Factor de Sostenibilidad y del Índice de Revalorización del sistema de pensiones de la seguridad social: régimen jurídico», Relaciones Laborales, n. ${ }^{\circ}$ 5, (2014): 115. 